\title{
Focal Podocyte Injury
}

National Cancer Institute

\section{Source}

National Cancer Institute. Focal Podocyte Injury. NCI Thesaurus. Code C82969.

A morphologic finding indicating focal damage or loss of the podocytes in the renal tissue. 